\title{
Molecular serum signature of treatment resistant depression
}

Tillmann Ruland M.D. 1,3*\#, Man K Chan Ph.D. ${ }^{*}$, Pawel Stocki Ph.D. ${ }^{3}$, Laura Grosse MSc. ${ }^{1,2}$, Matthias Rothermundt M.D. ${ }^{1}$, Jason D Cooper Ph.D. ${ }^{3}$, Volker Arolt M.D. ${ }^{1}$, Sabine Bahn M.D., Ph.D. ${ }^{3}$

\footnotetext{
${ }^{1}$ Mood and Anxiety Disorders Research Unit, Department of Psychiatry and Psychotherapy, University of Muenster, Germany

${ }^{2}$ Radiology Morphological Solutions, Rotterdam, The Netherlands

${ }^{3}$ Department of Chemical Engineering and Biotechnology, University of Cambridge, Cambridge, UK
}

${ }^{*}$ Both authors contributed equally to this work and should be considered shared first authors.

\#Corresponding author Tillmann.Ruland@ukmuenster.de , Tel.: 00492518356601

\section{Conflict of Interest}

Prof. Bahn is a director of Psynova Neurotech Ltd. Dr. Cooper is a consultant for Psynova Neurotech Ltd. No other authors report potential conflict of interest.

\section{Acknowledgments}

This study was supported by EU-FP7-HEALTH-F2-2008-222963 "MOODINFLAME" and by EU-FP7-PEOPLE-2009-IAPP "PSYCH-AID". These supporters had no further role in study design, in the collection, analysis and interpretation of data, in the writing of the report, and in the decision to submit the paper for publication.

Drs Chan, Cooper and Prof. Bahn were also supported by grants from the Stanley Medical Research Institute (no. 07R-1888) and the EU-FP7 SchizDX. 


\section{ABSTRACT}

\section{Rationale}

A substantial number of patients suffering from major depressive disorder (MDD) do not respond to multiple trials of antidepressants, develop a chronic course of disease and become treatment resistant. Most of the studies investigating molecular changes in treatment resistant depression (TRD) have only examined a limited number of molecules and genes. Consequently, biomarkers associated with TRD are still lacking.

\section{Objectives}

To use recently advanced high-throughput proteomic platforms to identify peripheral biomarkers of TRD defined by two staging models, the Thase and Rush staging model (TRM) and the Maudsley Staging Model (MSM).

\section{Methods}

Serum collected from an inpatient cohort of 65 individuals suffering from MDD was analysed using two different mass spectrometric-based platforms, label-free liquid chromatography mass spectrometry (LC-MSE) and selective reaction monitoring (SRM), as well as a multiplex bead based assay.

\section{Results}

In the LC-MSE analysis proteins involved in acute phase response, complement activation and coagulation were significantly different between the staging groups in both models. In the multiplex bead based assay analysis TNF- $\alpha$ levels (log(odds)=-4.95, $\mathrm{p}=0.045$ ) were significantly different in the TRM comparison.

Using SRM significant changes of three apolipoproteins $A-I(\beta=0.029, p=0.035), M(\beta=-$ 0.017, $\mathrm{p}=0.009)$ and $\mathrm{F}(\beta=-0.031, \mathrm{p}=0.024)$ were associated with the TRM but not the MSM.

\section{Conclusion}

Overall, our findings suggest that proteins, which are involved in immune and complement activation, may represent potential biomarkers that could be used by clinicians to identify high-risk patients. Nevertheless, given that the molecular changes between the staging groups were subtle, the results need to be interpreted cautiously. 
Keywords: treatment resistant depression, mass spectrometry, staging, serum 


\section{INTRODUCTION}

The goal of treatment in major depressive disorder (MDD) is complete remission (Keller 2003). Especially in the treatment of severely ill patients, antidepressant pharmacotherapy is one of the key strategies. However, after the initial trial of antidepressant treatment with selective serotonin reuptake inhibitors (SSRI) remission can only be achieved in $30 \%$ of patients (Rush et al. 2006). Furthermore, a substantial number of patients do not respond to multiple trials of antidepressants and develop a chronic course of disease and become treatment resistant. Chronically depressed patients have a significant quality-of-life impairment, and a chronic course of disease is associated with a high socioeconomic burden (Rapaport et al. 2005). In order to assess resistant patients during the course of antidepressant treatment, different staging models have been proposed. These models vary considerably in definition and measurement of treatment resistant depression (TRD) (Fava 2003; Ruhé et al. 2012; McIntyre et al. 2014; Trevino et al. 2014). In addition, none of the existing models are routinely used by clinicians.

Initially, Thase and Rush introduced a simple staging system for staging antidepressant resistance, the Thase and Rush Model (TRM), aimed at clinical psychiatrists managing non-responders (Thase and Rush 1997). The model consists of five stages usually beginning with a SSRI as first line intervention. The model has been used and reviewed extensively (Fava 2003; Nemeroff 2007; Ruhé et al. 2012). Another approach, the Maudsley Staging Model (MSM), which was proposed by Fekadu and colleagues (2009), attempted to overcome some disadvantages of the hierarchical Thase and Rush model, for example, by incorporating electroconvulsive therapy (ECT) as a non-hierarchical item (Fekadu et al. 2009). Furthermore, the authors tried to take into account both severity of disease and duration of illness (see Supplementary Figure 3).

Other staging models such as the European Staging Model or the Massachusetts General Hospital Staging Model were not analysed in the present study. These models propose a minimum trial duration of 6 weeks, whereas Thase and Rush originally proposed a 4week trial duration as minimum trial length. The national and international guidelines recommend treatment modification after 4 weeks of treatment without response (NCCMH 2010; Deutsche Gesellschaft für Psychiatrie 2015). Therefore the adequate trial 
length for a treatment trial without response was considered to be 4 weeks in our study. Furthermore, compared to the European Staging Model and the Massachusetts General Hospital Staging Model only the MSM includes both duration of illness and severity of depression (Ruhé et al. 2012).

In the absence of biomarkers, both staging models addressed treatment resistant depression solely on the basis of clinical information. In order to sharpen our diagnostic assessment and to individually target our treatment strategies, there is an unmet need for valid blood-based biomarkers (Chan et al. 2014; Niculescu et al. 2015; Bahn and Chan 2015).

Several studies have investigated molecules and genes associated with treatment response as well as TRD. Catechol-0-methyltransferase (COMT), brain-derived neurotrophic factor (BDNF) and the serotonin transporter (SLC6A4) gene polymorphisms have been investigated and may be associated with TRD and treatment response (Baune et al. 2008; Schosser et al. 2012). However, analysis of other candidates, for example cyclic adenosine monophosphate response element binding (CREB1) or dystrobrevin binding protein 1 (DTNBP1) gene, have not shown significant association with TRD (Schosser et al. 2012). Furthermore, a recently published large genome wide association study investigating genetic variation that may contribute to response to SSRI treatment has failed to show any significance at the genome-wide level (Biernacka et al. 2015).

Cytokines and other proteins involved in inflammation such as Interleukin 6 (IL-6) and tumor necrosis factor (TNF) may be implicated in the response to treatment with antidepressants and the development of TRD (Lanquillon et al. 2000; O'Brien et al. 2007; Powell et al. 2013). A possible role of TNF in TRD has even led to clinical trials investigating the effect of TNF-alpha antagonists such as infliximab as a monotherapy or an add on medication in patients suffering from TRD (Raison et al. 2013; Schmidt et al. 2014). In summary, until now molecular markers enhancing clinical staging or a blood test for response prediction or the identification of patients at high-risk of developing TRD are lacking. 
Apart from the above-mentioned methods, high throughput proteomic techniques such as mass spectrometry may offer an alternative to discover blood-based protein biomarkers for TRD and treatment response (Chan et al. 2014). Moreover, a recently developed mass spectrometric analysis method, which makes use of triple quadrupole mass spectrometry, known as selective reaction monitoring (SRM) provides a new tool for the quantitative and highly specific detection of pre-selected analytes in complex biological samples such as human serum (Lange et al. 2008; Picotti and Aebersold 2012). With this in mind and to extend the previous work on TRD biomarkers, we set out to identify serum biomarkers of TRD using different high throughput proteomic platforms. We hypothesized that molecular changes would be detectable across the different staging groups and tested this hypothesis by comparing two clinical models of treatment resistant depression, the TRM and the MSM, and using an inpatient cohort of 65 individuals suffering from TRD.

\section{MATERIALS AND METHODS}

\section{Study participants and sample collection}

This study was approved by the ethics committee of the medical association WestphaliaLippe, Germany (reference 2009-019-f-S). After study procedures had been fully explained, subjects provided written informed consent.

Sixty-five patients with detailed clinical information were selected from a cohort recruited for the EU funded MoodInflame project aimed at early diagnosis, treatment and prevention of mood disorders targeting the activated inflammatory response system (reference: 222963) (for more information see http://moodinflame.eu). All patients were MDD inpatients from three different centres, which were diagnosed with a major depressive disorder and were taking antidepressant medication at the time of sample collection. Patients were screened and included at any time during their inhospital treatment. All patients met the Diagnostic and Statistical Manual of Mental Disorders, 4th Edition, Text Revision (DSM-IV-TR) criteria for MDD and clinical tests including administration of the Inventory of Depressive Symptomatology (IDS- $\mathrm{C}_{30}$ ) assessment were performed by psychiatrists under good clinical practice-compliance to minimise variability at inclusion. All patients were symptomatic at inclusion (ICD-C 30 
>12). MDD patients with other psychiatric co-morbidities were identified and screened for personal or family history of neuropsychiatric disorders using M.I.N.I. (German Version 5.0.0). Remission was defined as an IDS-C of $\leq 11$ at discharge and partial remission defined as IDS-C of $\geq 11$ at discharge (Trivedi et al. 2004; Huijbers et al. 2012).

\section{Staging of patients}

All patients were staged using the Thase and Rush Model (TRM) as well as Maudsley Staging Model (MSM). For the TRM, patients were strictly staged according to definitions of the stages in the original publication by an experienced clinician (see Supplementary Figure 1) (Thase and Rush 1997). An adequate treatment trial was defined as a period of at least four weeks with a moderate dose in line with national guidelines. Only clinical information concerning the current episode was used for the TRM and MSM. This included a period of eight weeks before admission for which reliable data of medication compliance could be collected for every patient. MSM stages were assigned based on the total score meaning mild (scores $=3-6)$, moderate $($ scores $=7-10)$ and severe $($ scores $=$ 11-15) treatment resistance (see Supplementary Figure 2) (Fekadu et al. 2009). In general, medication only received a score if it was given at adequate dose for at least six weeks. Therefore some patients treated with a tricyclic antidepressants (TCA) or electroconvulsive therapy (ECT) at the time of blood collection did not receive a score because trial length of the TCA or previous antidepressants were not sufficient for classification purposes.

Since only one patient reached stage III resistance with a score of 11 , the patient was included in the group of patients with stage II resistance (not being an outlier in statistical analysis). Important patient demographic and clinical characteristics such as gender, age, BMI, smoking alcohol consumption, chronic illness, family psychiatric disorder, depression severity (IDS-C) and use of non-psychiatric medication as well as psychiatric comorbidities were compared for both models in order to detect significant differences between the staging groups. 


\section{Liquid chromatography coupled tandem mass spectrometry (LC-MSE ${ }^{\mathrm{C}}$ analysis}

Serum samples were randomized and quality controls were added, before depleting of the most abundant proteins using a MARS14 (Agilent, USA) on a ÄKTA ${ }^{\mathrm{TM}}$ purifier UPC 10 chromatography system (GE Healthcare, UK) as described previously (Jaros et al. 2013).

A tryptic digest was performed after depletion and the samples were stored at $-80{ }^{\circ} \mathrm{C}$ until LC-MSE analysis was performed. Using a Waters quadrupole time of flight (QTof) Premier mass spectrometer LC-MSE analysis was carried out by running every sample in triplicate, as described by Levin et al. After ProteinLync Global Server v.2.5. (Waters Corporation) and Rosetta Inpharmatics Biosoftware Elucidator v.3.3 (USA) data processing protein identification was performed as described previously (Stelzhammer et al. 2014).

\section{Selective reaction monitoring (SRM) mass spectrometry}

Samples were analysed by using a targeted SRM mass spectrometry approach on a predetermined set of peptides as described previously (Lange et al. 2008; Gottschalk et al. 2014). Briefly a Xevo TQ-S mass spectrometer (Waters Corporation) was coupled online through a New Objective nanoESI emitter ( $7 \mathrm{~cm}$ length, $10 \mathrm{~mm}$ tip; New Objective) to a nanoAcquity nano-ultra-performance liquid chromatography system (Waters Corporation). Peptide selection was done for candidate proteins identified previously by our lab and others (see Supplementary Table 1) (Penninx et al. 2003; Hummel et al. 2011; Stelzhammer et al. 2014). For each target peptide, a heavy isotope-labeled internal standard (JPT Peptide Technologies $\mathrm{GmbH}$ ) was spiked in the peptide mixture for accurate quantification and identification.

Transitions were calculated and selected using Skyline version v2.5 (MacLean et al. 2010). Each transition corresponded to singly charged y-ions from doubly- or triplycharged precursor ions in the range of 350-1250Da. Method refinement was performed on quality control samples in order to select for the peptides with the maximal intensities and highest spectral library similarity (dotp > 0.9). A further development step of analyzing heavy-label spiked quality control samples in scheduled SRM mode was used to confirm identity via co-elution, extract the optimal fragment ions for SRM analysis, obtain accurate peptide retention times, and optimize collision energy and 
cone voltage for the quantification run applying Skyline software (MacCoss Lab Software; MacLean et al., 2010).

\section{Multiplex bead based immunoassay}

High Sensitivity Hu Cytokine-T cell (MAGPX10223002) from Millipore was used as described in the manufacturers protocol. In summary, samples were thawed and antibody-immobilized beads were prepared. After preparing standards and buffers, the plate was prepared by washing it with a wash buffer. Samples, Standards and Beads were added to the appropriate wells and incubated overnight at $4^{\circ} \mathrm{C}$. Contents were then removed and detection antibodies added. Following a 1 hour incubation at room temperature (RT) Streptavidin-Phycoerythrin was added and incubated for another 30 minutes at RT. Finally drive fluid was added and the plate was run on a Luminex MagPix Plate Reader.

\section{Statistics}

All statistical analyses were performed in $\mathrm{R}$ (http://www.R-project.org/) (R Development Core Team 2013).

\section{LC-MS ${ }^{\mathrm{E}}$ and SRM data analysis}

The processed and normalised LC-MSE data was log transformed to stabilize variance and quality controls (QC) were assessed. Peptides with over 30\% missing values and missed cleavage were excluded. Sample outliers were examined using principal components analysis (PCA) (Beniger et al. 1980) and through inspection of quantilequantile (Q-Q) plots.

The SRM data was pre-processed using the R package MSstats (Clough et al. 2012). The data were $\log _{2}$ transformed and quantile normalisation was applied to remove systematic bias between MS runs. The resulting profile, QC and condition plots were carefully inspected to identify potential sources of variation for each protein, evaluate any systematic bias between MS runs and assess the variability of each condition per protein, respectively. Transitions with over 30\% missing values were excluded. Sample outliers were examined as described above. 
For both the LC-MSE and SRM data, protein-level quantification and testing for differential abundance between the staging groups were performed using the random intercept linear mixed-effects model, as implemented in the R package nlme (Pinheiro et al. 2009). Random intercepts for subjects nested within recruitment centres were specified for each model to account for hierarchical structure of the data. The confounding effects of patient demographic characteristics such as age, BMI, gender, smoking status, alcohol use and chronic illnesses were accounted for in each model (fixed effects). False discovery rate was controlled according to Benjamini and Hochberg (Benjamini and Hochberg 1995).

\section{Multiplex bead-based immunoassay data analysis}

Immunoassay data were pre-processed to remove analytes with greater than $30 \%$ missing values. Missing values are defined as analytes with measurement values below or above the detection limits. The resulting data were $\log _{10}$ transformed. Logistic regression was applied with staging status as the outcome and analyte as the predictor variables. The demographic variables listed above were made available for forward and backward stepwise selection (Hastie, T.J. Pregibon, D. 1992), with selection based upon Bayesian Information Criteria (BIC) (Schwarz 1978).

\section{RESULTS}

Demographic and clinical variables for the staging groups in both models were compared (Table 1). For the TRM, no significant differences existed across all covariates including age, gender, BMI, smoking and depression severity. For the MSM only depression severity was significantly different between stage I (mean $=30.53$, standard deviation $= \pm 8.15$ ) and stage II (mean $=41.72$, standard deviation \pm 9.50 ), which was due to depression severity being an item of the model itself (see Supplement Figure 2).

Using logistic regression, both models showed no significant predictive capability to discriminate between the group of patients achieving remission and the patients with only partial remission at discharge (for TRM $\log$ (odds)=-0.89, p=0.119; for MSM $\log ($ odds $)=-1.16, p=0.067)$. Although, the predictive validity of the MSM has been shown previously, this could not be replicated in our study. 
Using a non-hypothesis driven label-free LC-MSE approach, a number of proteins were identified to be significantly different between the staging groups in both models (Table 2, Supplementary Table 2 for the list of all detected proteins). Based on a Gene Ontology (GO) term analysis these proteins were mainly involved in the biological process of acute phase response, complement activation, coagulation and oxygen transport (The Gene Ontology Consortium 2000; The Gene Ontology Consortium 2015). For the TRM stage comparison, a total of eight proteins could be detected as significantly different: Serum amyloid P-component $(\beta=0.035, p=0.008 \mathrm{FC}=1.08)$, Ficolin-3 $(\beta=0.033$, $\mathrm{p}=0.021 \mathrm{FC}=1.08), \mathrm{C} 4 \mathrm{~b}-\mathrm{binding}$ protein beta chain $(\beta=0.053, \mathrm{p}=0.023, \mathrm{FC}=1.13), \mathrm{C} 4 \mathrm{~b}-$ binding protein alpha chain $(\beta=0.030, p=0.024, \mathrm{FC}=1.07)$, Complement $\mathrm{C} 1 \mathrm{q}$ subcomponent subunit $C(\beta=0.026, p=0.037, F C=1.06)$, Histidine-rich glycoprotein $(\beta=-$ 0.053, $\mathrm{p}=0.024, \mathrm{FC}=0.88)$, Nuclear factor of activated T-cells $(\beta=0.047, \mathrm{p}=0.027$, $\mathrm{FC}=1.11)$ and Beta-Ala-His dipeptidase $(\beta=-0.053, \mathrm{p}=0.049, \mathrm{FC}=0.88)$. Interestingly, almost all of the proteins were involved in acute phase response, complement activation and coagulation.

For the MSM stage comparison, ten proteins were significantly changed: Heparin cofactor $2(\beta=-0.023, p=0.004, F C=0.95)$, Plasma serine protease inhibitor $(\beta=0.034$, $\mathrm{p}=0.012, \quad \mathrm{FC}=1.08)$, Antithrombin-III $(\beta=0.039, \mathrm{p}=0.023, \mathrm{FC}=1.09)$, Interleukin-1 receptor accessory protein $(\beta=0.067, p=0.026, F C=1.17)$, Complement factor $\mathrm{D}(\beta=0.059$, $\mathrm{p}=0.037, \quad \mathrm{FC}=1.15)$, Hemoglobin subunit alpha $(\beta=-0.400, \mathrm{p}=0.008, \quad \mathrm{FC}=0.40)$, Hemoglobin subunit beta $(\beta=-0.227, \mathrm{p}=0.017, \mathrm{FC}=0.59)$, Putative postmeiotic segregation increased 2-like protein 11 ( $\beta=0.099, \mathrm{p}=0.004, \mathrm{FC}=1.26)$, Calcium-binding protein $5(\beta=-0.068, p=0.023, F C=0.85)$, Cytosolic beta-glucosidase $(\beta=0.051, p=0.039$, $\mathrm{FC}=1.12)$.

On a protein-level, no overlap could be detected between MSM and TRM stage comparisons. However, the top GO terms were similar in both comparisons. Except for the proteins Hemoglobin subunit alpha and beta involved in oxygen transport, fold changes were mainly subtle. Testing for multiple corrections using the Benjamini Hochberg method yielded no statistically significant results (adjusted p-value $>0.05$ ). 
A multiplex bead based assay was used to further elucidate possible changes in inflammatory proteins previously implicated in depression (Penninx et al. 2003; Kaestner et al. 2005; Simon et al. 2008). A total of 7 analytes were included in the analysis (IFN- $\gamma$, IL12p70, IL-4, IL-6, IL-7, IL-8, TNF- $\alpha$ ). In the TRM comparison, significant differences in TNF- $\alpha$ levels between the groups could be detected $(\log ($ odds $)=-4.95, \mathrm{p}=0.045)$. There were no significant differences for the MSM stage comparison (Table 3). In addition, no significant correlation could be shown between the MSM score and the analytes (data not shown).

Furthermore, another mass spectrometry method, Selective Reaction Monitoring (SRM), was used to analyse the samples. For the SRM assay a panel of apolipoproteins and inflammation-related proteins implicated in major depression was selected (see Supplementary Table 1). These analytes were chosen based on previous findings by our group (Stelzhammer et al. 2014; Bot et al. 2015) and others (Hummel et al., 2011;). In addition, the most significant proteins identified by label-free LC-MSE ${ }^{E}$ were selected for further validation. Significant changes could only be detected for the TRM stage comparison (Table 4). Three apolipoproteins A-I $(\beta=0.029, p=0.035, F C=1.02), M(\beta=-$ 0.017, $\mathrm{p}=0.009, \mathrm{FC}=0.99)$ and $\mathrm{F}(\beta=-0.031, \mathrm{p}=0.024, \mathrm{FC}=0.98)$ as well as alpha-1antichymotrypsin $(\beta=0.025, p=0.032, F C=1.02)$ were found to be changed. However, changes of Ficolin-3, Complement $\mathrm{C} 1 \mathrm{q}$ subcomponent subunit $\mathrm{C}$ and Histidine-rich glycoprotein, which were significantly changed in the label-free LC-MSE experiment, could not be validated by SRM.

\section{DISCUSSION}

The objective of this study was to evaluate possible molecular phenotypes underlying treatment resistant depression based on two clinical staging methods using proteomics. The use of proteomics for discovering blood-based biomarkers in different medical fields has progressed substantially over the last years (Hanash et al. 2008; Shao et al. 2015). New analysis methods such as SRM have been developed recently and offer novel ways of analysing a predetermined set of proteins in a complex mixture like human serum across multiple samples (Picotti and Aebersold 2012). To our knowledge, this is 
the first study using SRM to detect pre-selected analytes in a MDD cohort suffering from TRD.

While group comparison in the TRM showed significantly changed proteins in all three assays, only the LC-MSE analysis showed significant changes in the MSM. GO Term analysis revealed that the identified proteins were mainly involved in complement activation and coagulation. Upregulation of the closely interacting proteins serum amyloid P component and the C4b-binding protein in the Stage II TRM group might suggest an altered regulation of the complement system in more severely affected patients (García de Frutos and Dahlbäck 1994). Interestingly, changes of TNF alpha levels in the TRM model using a multiplex bead based immunoassay corresponded to results of previous studies, which showed a relationship between response and TNF alpha level decrease (Lanquillon et al. 2000; Strawbridge et al. 2015). SRM analysis revealed three apolipoproteins being changed in the TRM group comparison. A possible role of apolipoprotein changes during treatment of MDD has been suggested by previous studies (Sadeghi et al. 2011; Hummel et al. 2011).

A possible role of inflammation in the pathogenesis of MDD has been studied extensively in patients as well as in animal models of depression (Dantzer et al. 2008; Iwata et al. 2013). Furthermore, levels of inflammation related genes predict lack of response to antidepressants (Cattaneo et al. 2013). A recent meta-analysis revealed an association of IL-1 $\beta$ and IL-6 levels with suicidality (Black and Miller 2015). Anti-inflammatory drugs have also been found to antagonize the therapeutic efficacy of antidepressant agents (Warner-Schmidt et al. 2011; Miller and Raison 2016). Therefore, further studies are needed to elucidate a potential relationship between inflammatory activation and different stages of TRD.

Some limitations of the study design have to be considered. The sample size for this pilot study was small and an independent validation cohort was not available, hence larger prospective studies are warranted. Furthermore, the cohort of patients used in this study was not specifically recruited to assess all stages of treatment resistant depression as defined by the two staging methods. Therefore, patients with a very high number of unsuccessful treatment trials (five or more) could be underrepresented. Since the cohort did not include outpatients, no control group of responders to a first treatment trial was 
available. In addition, the TRM did not account for the use of augmentation strategies. Since medication had to be taken for a period of six weeks in MSM, only 16 patients received augmentation therapy long enough to score $>0$. Robust clinical data offering information about response time to an augmentation strategy in TRD is not available and needs further evaluation in specifically designed clinical trials (Keller 2005; Carvalho et al. 2007). Other staging models have been designed, which were not analysed in this study, therefore leaving the question open if different molecular phenotypes underlying the staging in these models (Ruhé et al. 2012).

In conclusion, our findings suggest that proteins involved in complement system activation, inflammatory response, and lipid transport could be interesting candidates to stratify TRD at the molecular level. However, given that the molecular changes between the staging groups were subtle, the results have to be interpreted cautiously. With regard to the limitations of study, this pilot data shows the need for optimization of the clinical staging models by conducting prospective clinical trials. Advances in proteomics technologies in terms of analytical sensitivity and resolution as well as costeffectiveness now allow for improved targeted molecular measurements. Such advances may offer a wider range of biomarkers potentially capable of allowing for stratification of molecular phenotypes underlying treatment resistant depression. 


\section{CONTRIBUTORS}

TR and MKC designed the study, are responsible for the statistical analyses and the first draft of the manuscript. VA, MR, LS, TR were responsible for recruitment and clinical characterization of the patients, TR and PS performed the laboratory work. JC supervised the statistical analyses. VA and SB supervised the study and were involved in the design of the study. All authors contributed to and have approved the final manuscript.

\section{CONFLICT OF INTEREST}

Prof. Bahn is a director of Psynova Neurotech Ltd. Dr. Cooper is a consultant for Psynova Neurotech Ltd. No other authors report potential conflict of interest.

\section{ACKNOWLEDGMENTS}

This study was supported by EU-FP7-HEALTH-F2-2008-222963 “MOODINFLAME” and by EU-FP7-PEOPLE-2009-IAPP "PSYCH-AID”. These supporters had no further role in study design, in the collection, analysis and interpretation of data, in the writing of the report, and in the decision to submit the paper for publication.

Drs Chan, Cooper and Prof. Bahn were also supported by grants from the Stanley Medical Research Institute (no. 07R-1888) and the EU-FP7 SchizDX. 
TABLE 1

\begin{tabular}{|c|c|c|c|c|c|c|}
\hline & \multicolumn{3}{|c|}{ Thase and Rush staging model (TRM) } & \multicolumn{3}{|c|}{ Maudsley Staging Model (MSM) } \\
\hline & Stage I & Stage II & P-value & Stage I & Stage II & P-value \\
\hline $\mathbf{n}$ & 36 & 29 & NA & 41 & 24 & NA \\
\hline Gender $(M / F)^{F}$ & $(15 / 21)$ & $(18 / 11)$ & 0.136 & $(23 / 18)$ & $(10 / 14)$ & 0.310 \\
\hline Age $^{W}$ & $39.69( \pm 12.60)$ & $40.30( \pm 11.12)$ & 0.979 & $41.43( \pm 11.74)$ & $36.71( \pm 11.79)$ & 0.138 \\
\hline BMIW & $27.83( \pm 5.88)$ & $27.07( \pm 4.96)$ & 0.853 & $27.36( \pm 5.98)$ & $28.25( \pm 4.81)$ & 0.215 \\
\hline Smoking (yes/no) ${ }^{\mathrm{F}}$ & $(13 / 23)$ & $(9 / 20)$ & 0.794 & $(14 / 27)$ & $(8 / 16)$ & 1.000 \\
\hline Alcohol (yes/no) ${ }^{\mathrm{F}}$ & $(30 / 15)$ & $(16 / 20)$ & 0.324 & $(18 / 23)$ & $(13 / 11)$ & 0.452 \\
\hline $\begin{array}{l}\text { Chronic illness } \\
\text { (yes/no) }\end{array}$ & $(6 / 30)$ & $(9 / 20)$ & 0.238 & $(9 / 32)$ & $(6 / 18)$ & 0.770 \\
\hline $\begin{array}{l}\text { Family psychiatric } \\
\text { disorder (yes/no/NA) }\end{array}$ & $(25 / 7 / 4)$ & $(17 / 9 / 3)$ & 0.558 & $(29 / 10 / 2)$ & $(13 / 6 / 5)$ & 0.129 \\
\hline $\begin{array}{l}\text { Depression severity } \\
\text { (IDS-C30) }\end{array}$ & $33.53( \pm 9.56)$ & $33.18( \pm 9.56)$ & 0.372 & $30.53( \pm 8.15)$ & $41.72( \pm 9.50)$ & $2.0 \mathrm{E}-06$ \\
\hline $\begin{array}{l}\text { Time (weeks) between } \\
\text { admission \& discharge }\end{array}$ & $9.84( \pm 5.95)$ & $10.79( \pm 7.60)$ & 0.50 & $9.45( \pm 4.90)$ & $11.65( \pm 8.93)$ & 0.51 \\
\hline $\begin{array}{l}\text { Use of non-psychiatric } \\
\text { medication (yes/no) }\end{array}$ & $(11 / 25)$ & $(11 / 18)$ & 0.603 & $(11 / 30)$ & $(11 / 13)$ & 0.174 \\
\hline $\begin{array}{l}\text { Psychiatric } \\
\text { Comorbidities (yes/no) }\end{array}$ & $(12 / 24)$ & $(10 / 19)$ & 1.000 & $(14 / 27)$ & $(8 / 16)$ & 1.000 \\
\hline $\begin{array}{l}\text { Remission/Partial } \\
\text { Remission }^{F}\end{array}$ & $(14 / 22)$ & $(6 / 23)$ & $\mathrm{NA}^{*}$ & $(16 / 25)$ & $(4 / 20)$ & $\mathrm{NA}^{*}$ \\
\hline
\end{tabular}

$\mathbf{F}=$ Fisher's exact test; $\mathbf{W}=$ Wilcoxon test; $\mathbf{T R M}=$ Thase and Rush staging model; $\mathbf{M S M}=$ Maudsley Staging Model; $\mathbf{n}$ = sample size; $\mathbf{B M I}=$ Body Mass Index; $\mathbf{I D S}-\mathbf{C}_{\mathbf{3 0}}=$ Inventory of Depressive Symptomatology IDS$\mathrm{C}_{30}$

Legend to table 1: Patient demographic and clinical characteristics. The staging groups are shown separately for TRM and MSM stages (mean \pm sd for continuous variables) significance level at $p<$ 0.05. * Using logistic regression, both models showed no significant predictive capability (see results section for details).

\section{TABLE 2}

TRM

\begin{tabular}{llcccc}
\hline $\begin{array}{l}\text { GO Term } \\
\text { Biological progress }\end{array}$ & Protein names & $\boldsymbol{\beta}$ & $\begin{array}{c}\text { Std. } \\
\text { Error }\end{array}$ & P-value & FC (TRM II /TRM I) \\
\hline Acute phase response & Serum amyloid P-component & 0.035 & 0.013 & 0.008 & 1.08 \\
\hline $\begin{array}{l}\text { Complement } \\
\text { activitaion }\end{array}$ & Ficolin-3 & 0.033 & 0.014 & 0.021 & 1.08 \\
& C4b-binding protein beta chain & 0.053 & 0.023 & 0.023 & 1.13 \\
& C4b-binding protein alpha chain & 0.030 & 0.013 & 0.024 & 1.07 \\
\hline Blood coagulation & Complement C1q subcomponent subunit C & 0.026 & 0.012 & 0.037 & 1.06 \\
\hline Immune response & Nuclear factor of activated T-cells & -0.053 & 0.023 & 0.024 & 0.88 \\
\hline Dipetidase & Beta-Ala-His dipeptidase & 0.047 & 0.021 & 0.027 & 1.11 \\
\hline & & -0.053 & 0.026 & 0.049 & 0.88 \\
\hline MRM & & & & & \\
\hline GO Term & Protein names & & & & \\
Biological progress & Heparin cofactor 2 & $\boldsymbol{\beta}$ & Std. & \multirow{2}{*}{ P-value } & FC (MSM II/MSM I) \\
\hline Blood coagulation & Plasma serine protease inhibitor & -0.023 & 0.008 & 0.004 & 0.95 \\
& Antithrombin-III & 0.034 & 0.013 & 0.012 & 1.08 \\
\hline Immune response & Interleukin-1 receptor accessory protein & 0.039 & 0.017 & 0.023 & 1.09 \\
\hline Complement & Complement factor D & 0.067 & 0.029 & 0.026 & 1.17 \\
\hline activitation & Hemoglobin subunit alpha & 0.059 & 0.028 & 0.037 & 1.15 \\
\hline Oxygen transport & Hemoglobin subunit beta & -0.400 & 0.145 & 0.008 & 0.40 \\
\hline Mismatch repair & Putative postmeiotic segregation & -0.227 & 0.092 & 0.017 & 0.59 \\
\hline
\end{tabular}




\begin{tabular}{llcccc} 
& increased 2-like protein 11 & & & & \\
\hline Signal transduction & Calcium-binding protein 5 & -0.068 & 0.029 & 0.023 & 0.85 \\
\hline Glycosidase & Cytosolic beta-glucosidase & 0.051 & 0.024 & 0.039 & 1.12 \\
\hline
\end{tabular}

TRM = Thase and Rush staging model; MSM = Maudsley Staging Model; $\boldsymbol{\beta}=$ Regression Coefficient

Estimates; Std. Error = Regression standard error; $\mathbf{F C}=$ Fold change.

Legend to table 2: Results of the non-hypothesis driven label-free LC-MSE approach. (FC= fold change) Go Terms shown for the biological process in which the identified protein is involved. Significance level at $\mathrm{p}<0.05$

\section{TABLE 3}

\begin{tabular}{|l|c|c|c|}
\hline \multicolumn{1}{l|}{ TRM } & Log (odds) & Std. Error & P-value \\
\hline Protein & 0.58 & 1.01 & 0.568 \\
\hline IFN- $\boldsymbol{~}$ & 0.08 & 0.51 & 0.870 \\
\hline IL-10 & -0.61 & 0.68 & 0.365 \\
\hline IL-12p70 & -0.27 & 0.63 & 0.672 \\
\hline IL-2 & -0.03 & 0.47 & 0.957 \\
\hline IL-4 & -0.25 & 0.71 & 0.723 \\
\hline IL-6 & 0.64 & 1.39 & 0.644 \\
\hline IL-7 & 0.43 & 0.98 & 0.664 \\
\hline IL-8 & -4.95 & 2.47 & 0.045 \\
\hline TNF- $\alpha$ & & & \\
\hline
\end{tabular}

\begin{tabular}{|c|c|c|c|}
\hline \multicolumn{4}{|l|}{ MSM } \\
\hline Protein & Log (odds) & Std. Error & P-value \\
\hline IFN- $\gamma$ & 1.24 & 1.59 & 0.249 \\
\hline IL-10 & -0.24 & 0.64 & 0.646 \\
\hline IL-12p70 & 0.41 & 0.88 & 0.566 \\
\hline IL-2 & -0.01 & 0.80 & 0.987 \\
\hline IL-4 & -0.50 & 0.64 & 0.318 \\
\hline IL-6 & 0.32 & 0.85 & 0.669 \\
\hline IL-7 & 0.22 & 1.68 & 0.876 \\
\hline IL-8 & -0.81 & 1.26 & 0.445 \\
\hline TNF- $\alpha$ & -2.92 & 2.62 & 0.168 \\
\hline
\end{tabular}

TRM = Thase and Rush staging model; MSM = Maudsley Staging Model; Std. Error = Regression standard error; IFN- $\boldsymbol{\gamma}$ = Interferon-gamma; IL12p70 = Interleukin 12p70; IL-2 = Interleukin 2; IL-6 = Interleukin 6, IL-7 = Interleukin 7; IL-8 = Interleukin 8; TNF- $\alpha=$ tumor necrosis factor alpha

Legend table 3: Results of multiplex bead based assay. In TRM group comparison a significant difference was found for TNF- $\alpha$. No significant differences could be detected for MSM. Significance level at $p<0.05$.

TABLE 4

\begin{tabular}{l|cccc|cccc}
\hline & \multicolumn{4}{|c|}{ TRM } & \multicolumn{4}{c}{ MSM } \\
\hline Protein names & $\beta$ & $\begin{array}{c}\text { Std. } \\
\text { Error }\end{array}$ & P-value & $\begin{array}{c}\text { FC (TRM } \\
\text { II/TRM I) }\end{array}$ & $\beta$ & $\begin{array}{c}\text { Std. } \\
\text { Error }\end{array}$ & P-value & $\begin{array}{c}\text { FC (MSM } \\
\text { II/MSM I) }\end{array}$ \\
\hline Apolipoprotein M & -0.017 & 0.006 & 0.009 & 0.99 & 0.004 & 0.008 & 0.576 & 1.00 \\
Apolipoprotein F & -0.031 & 0.013 & 0.024 & 0.98 & -0.008 & 0.016 & 0.630 & 1.00 \\
Apolipoprotein A-I & 0.029 & 0.013 & 0.035 & 1.02 & -0.003 & 0.017 & 0.557 & 1.00 \\
Alpha-1-antichymotrypsin & 0.025 & 0.011 & 0.032 & 1.02 & 0.008 & 0.014 & 0.557 & 1.01 \\
\hline
\end{tabular}

TRM = Thase and Rush staging model; MSM = Maudsley Staging Model; $\beta=$ Regression Coefficient Estimates; Std. Error = Regression standard error; FC = Fold change 
Legend to table 4: Results of Multiple Reaction Monitoring analysis. No significant differences could be detected for MSM group comparison. ns. = not significant; significance level at $\mathbf{p}<0.05$.

\section{REFERENCES}

Bahn S, Chan MK (2015) What can we learn about depression from gene expression in peripheral tissues? Biol Psychiatry 77:207-9. doi: 10.1016/j.biopsych.2014.10.019

Baune BT, Hohoff C, Berger K, et al (2008) Association of the COMT val158met variant with antidepressant treatment response in major depression. Neuropsychopharmacology 33:924-32. doi: 10.1038/sj.npp.1301462

Beniger JR, Barnett V, Lewis T (1980) Outliers in Statistical Data. Contemp. Sociol. 9:560.

Benjamini Y, Hochberg Y (1995) Controlling the False Discovery Rate: A Practical and Powerful Approach to Multiple Testing. J R Stat Soc Ser B 57:289 - 300. doi: 10.2307/2346101

Biernacka JM, Sangkuhl K, Jenkins G, et al (2015) The International SSRI Pharmacogenomics Consortium (ISPC): a genome-wide association study of antidepressant treatment response. Transl Psychiatry 5:e553. doi: 10.1038/tp.2015.47

Black C, Miller BJ (2015) Meta-analysis of cytokines and chemokines in suicidality: Distinguishing suicidal versus nonsuicidal patients. Biol Psychiatry 78:28-37. doi: 10.1016/j.biopsych.2014.10.014

Bot M, Chan MK, Jansen R, et al (2015) Serum proteomic profiling of major depressive disorder. Transl Psychiatry 5:e599.

Carvalho AF, Cavalcante JL, Castelo MS, Lima MCO (2007) Augmentation strategies for treatment-resistant depression: A literature review. J. Clin. Pharm. Ther. 32:415-428.

Cattaneo A, Gennarelli M, Uher R, et al (2013) Candidate Genes Expression Profile Associated with Antidepressants Response in the GENDEP Study: Differentiating between Baseline / Predictors/' and Longitudinal / Targets/'. Neuropsychopharmacology 38:377-385.

Chan MK, Gottschalk MG, Haenisch F, et al (2014) Applications of blood-based protein biomarker strategies in the study of psychiatric disorders. Prog Neurobiol 122C:45-72. doi: 10.1016/j.pneurobio.2014.08.002

Clough T, Thaminy S, Ragg S, et al (2012) Statistical protein quantification and significance analysis in label-free LC-MS experiments with complex designs. BMC Bioinformatics 13 Suppl 1:S6. doi: 10.1186/1471-2105-13-S16-S6

Dantzer R, O'Connor JC, Freund GG, et al (2008) From inflammation to sickness and depression: when the immune system subjugates the brain. Nat Rev Neurosci 9:46-56. doi: 10.1038/nrn2297

Deutsche Gesellschaft für Psychiatrie P und N (DGPPN) (2015) S3-Leitlinie/Nationale Versorgungsleitlinie Unipolare Depression Langfassung. Ärztliches Zent für Qual der Medizin 1-264. doi: 10.6101/AZQ/000239

Fava M (2003) Diagnosis and definition of treatment-resistant depression. Biol Psychiatry 53:649-659. doi: 10.1016/S0006-3223(03)00231-2

Fekadu A, Wooderson S, Donaldson C, et al (2009) A multidimensional tool to quantify treatment resistance in depression: The Maudsley staging method. J. Clin. Psychiatry 70:177-184.

García de Frutos P, Dahlbäck B (1994) Interaction between serum amyloid P component and C4b-binding protein associated with inhibition of factor I-mediated C4b degradation. J Immunol 152:2430-2437.

Gottschalk MG, Wesseling H, Guest PC, Bahn S (2014) Proteomic enrichment analysis of psychotic and affective disorders reveals common signatures in presynaptic glutamatergic signaling and energy metabolism. Int J Neuropsychopharmacol 18:pyu019. doi: 10.1093/ijnp/pyu019

Hanash SM, Pitteri SJ, Faca VM (2008) Mining the plasma proteome for cancer biomarkers. Nature 452:571-579. doi: 10.1038/nature06916

Hastie, T.J. Pregibon D (1992) Generalized linear models. In: Chambers JM, Hastie TJ (eds) Statistical Models in S. Pacific Grove, CA, USA.,

Huijbers MJ, Spijker J, Donders ART, et al (2012) Preventing relapse in recurrent depression using mindfulness-based cognitive therapy, antidepressant medication or the combination: trial design and protocol of the MOMENT study. BMC Psychiatry 12:1-11. doi: 10.1186/1471-244X-12-125

Hummel J, Westphal S, Weber-Hamann B, et al (2011) Serum lipoproteins improve after successful pharmacologic antidepressant treatment: a randomized open-label prospective trial. J Clin Psychiatry 72:885-91. doi: 10.4088/JCP.09m05853blu

Iwata M, Ota KT, Duman RS (2013) The inflammasome: pathways linking psychological stress, depression, and systemic illnesses. Brain Behav Immun 31:105-14. doi: 10.1016/j.bbi.2012.12.008

Jaros JAJ, Guest PC, Bahn S, Martins-de-Souza D (2013) Affinity depletion of plasma and serum for mass 
spectrometry-based proteome analysis. Methods Mol Biol 1002:1-11. doi: 10.1007/978-1-62703360-2_1

Kaestner F, Hettich M, Peters M, et al (2005) Different activation patterns of proinflammatory cytokines in melancholic and non-melancholic major depression are associated with HPA axis activity. J Affect Disord 87:305-311. doi: 10.1016/j.jad.2005.03.012

Keller MB (2003) Past, present, and future directions for defining optimal treatment outcome in depression: remission and beyond. JAMA 289:3152-3160. doi: 10.1001/jama.289.23.3152\r289/23/3152 [pii]

Keller MB (2005) Issues in treatment-resistant depression. J. Clin. Psychiatry 66:5-12.

Lange V, Picotti P, Domon B, Aebersold R (2008) Selected reaction monitoring for quantitative proteomics: a tutorial. Mol Syst Biol 4:222. doi: 10.1038/msb.2008.61

Lanquillon S, Krieg JC, Bening-Abu-Shach U, Vedder H (2000) Cytokine production and treatment response in major depressive disorder. Neuropsychopharmacology 22:370-9. doi: 10.1016/S0893133X(99)00134-7

MacLean B, Tomazela DM, Shulman N, et al (2010) Skyline: An open source document editor for creating and analyzing targeted proteomics experiments. Bioinformatics 26:966-968. doi: 10.1093/bioinformatics/btq054

McIntyre RS, Filteau M-J, Martin L, et al (2014) Treatment-resistant depression: definitions, review of the evidence, and algorithmic approach. J Affect Disord 156:1-7. doi: 10.1016/j.jad.2013.10.043

Miller AH, Raison CL (2016) The role of inflammation in depression: from evolutionary imperative to modern treatment target. Nat Rev Immunol 16:22-34. doi: 10.1038/nri.2015.5

NCCMH (2010) Depression: the Treatment and Management of Depression in Adults (Update).

Nemeroff CB (2007) Prevalence and management of treatment-resistant depression. J Clin Psychiatry 68:17-25.

Niculescu AB, Levey D, Le-Niculescu H, et al (2015) Psychiatric blood biomarkers: avoiding jumping to premature negative or positive conclusions. Mol Psychiatry 20:286-288. doi: 10.1038/mp.2014.180

O’Brien SM, Scully P, Fitzgerald P, et al (2007) Plasma cytokine profiles in depressed patients who fail to respond to selective serotonin reuptake inhibitor therapy. J Psychiatr Res 41:326-31. doi: 10.1016/j.jpsychires.2006.05.013

Penninx BWJH, Kritchevsky SB, Yaffe K, et al (2003) Inflammatory markers and depressed mood in older persons: Results from the health, aging and body composition study. Biol Psychiatry 54:566-572. doi: 10.1016/S0006-3223(02)01811-5

Picotti P, Aebersold R (2012) Selected reaction monitoring-based proteomics: workflows, potential, pitfalls and future directions. Nat Methods 9:555-66. doi: 10.1038/nmeth.2015

Pinheiro J, Bates D, DebRoy S, et al (2009) nlme: Linear and Nonlinear Mixed Effects Models.

Powell TR, Schalkwyk LC, Heffernan AL, et al (2013) Tumor necrosis factor and its targets in the inflammatory cytokine pathway are identified as putative transcriptomic biomarkers for escitalopram response. Eur Neuropsychopharmacol 23:1105-14. doi: 10.1016/j.euroneuro.2012.09.009

R Development Core Team (2013) R: A Language and Environment for Statistical Computing. R Found. Stat. Comput. Vienna Austria 0:\{ISBN\} 3-900051-07-0.

Raison CL, Rutherford RE, Woolwine BJ, et al (2013) A randomized controlled trial of the tumor necrosis factor antagonist infliximab for treatment-resistant depression: the role of baseline inflammatory biomarkers. JAMA psychiatry 70:31-41. doi: 10.1001/2013.jamapsychiatry.4

Rapaport MH, Clary C, Fayyad R, Endicott J (2005) Quality-of-life impairment in depressive and anxiety disorders. Am J Psychiatry 162:1171-1178. doi: 10.1176/appi.ajp.162.6.1171

Ruhé HG, Van Rooijen G, Spijker J, et al (2012) Staging methods for treatment resistant depression. A systematic review. J. Affect. Disord. 137:35-45.

Rush AJ, Trivedi MH, Wisniewski SR, et al (2006) Acute and longer-term outcomes in depressed outpatients requiring one or several treatment steps: A STAR*D report. Am J Psychiatry 163:19051917. doi: 10.1176/appi.ajp.163.11.1905

Sadeghi M, Roohafza H, Afshar H, et al (2011) Relationship between depression and apolipoproteins A and B: a case-control study. Clinics (Sao Paulo) 66:113-117. doi: 10.1590/S1807-59322011000100020

Schmidt FM, Kirkby KC, Himmerich H (2014) The TNF-alpha inhibitor etanercept as monotherapy in treatment-resistant depression - report of two cases. Psychiatr Danub 26:288-90.

Schosser A, Serretti A, Souery D, et al (2012) European Group for the Study of Resistant Depression (GSRD)--where have we gone so far: review of clinical and genetic findings. Eur Neuropsychopharmacol 22:453-68. doi: 10.1016/j.euroneuro.2012.02.006

Schwarz G (1978) Estimating the Dimension of a Model. Ann. Stat. 6:461-464.

Shao S, Guo T, Aebersold R (2015) Mass spectrometry-based proteomic quest for diabetes biomarkers. 
Biochim Biophys Acta 1854:519-527. doi: 10.1016/j.bbapap.2014.12.012

Simon NM, McNamara K, Chow CW, et al (2008) A detailed examination of cytokine abnormalities in Major Depressive Disorder. Eur Neuropsychopharmacol 18:230-233. doi: 10.1016/j.euroneuro.2007.06.004

Stelzhammer V, Haenisch F, Chan MK, et al (2014) Proteomic changes in serum of first onset, antidepressant drug-naïve major depression patients. Int J Neuropsychopharmacol 1-10. doi: $10.1017 /$ S1461145714000819

Strawbridge R, Arnone D, Danese A, et al (2015) Inflammation and clinical response to treatment in depression: A meta-analysis. Eur. Neuropsychopharmacol. 25:1532-1543.

Thase ME, Rush AJ (1997) When at first you don't succeed: Sequential strategies for antidepressant nonresponders. In: Journal of Clinical Psychiatry. pp 23-29

The Gene Ontology Consortium (2000) Gene Ontology: tool for the unification of biology. Nat Genet 25:2529. doi: $10.1038 / 75556$

The Gene Ontology Consortium (2015) Gene Ontology Consortium: going forward. Nucleic Acids Res 43:D1049-D1056. doi: 10.1093/nar/gku1179

Trevino K, McClintock SM, McDonald Fischer N, et al (2014) Defining treatment-resistant depression: A comprehensive review of the literature. Ann Clin Psychiatry 26:222-32.

Trivedi MH, Rush AJ, Ibrahim HM, et al (2004) The Inventory of Depressive Symptomatology, Clinician Rating (IDS-C) and Self-Report (IDS-SR), and the Quick Inventory of Depressive Symptomatology, Clinician Rating (QIDS-C) and Self-Report (QIDS-SR) in public sector patients with mood disorders: a psych. Psychol Med 34:73-82.

Warner-Schmidt JL, Vanover KE, Chen EY, et al (2011) Antidepressant effects of selective serotonin reuptake inhibitors (SSRIs) are attenuated by antiinflammatory drugs in mice and humans. Proc Natl Acad Sci U S A 108:9262-7. doi: 10.1073/pnas.1104836108 